\title{
The Impact of Cultural Competition on Regional Economic Growth ----An Analysis based on Dynamic Spatial Panel
}

\author{
D.F. MENG \\ School of Economics and Business Administration, Chongqing University, China
}

\author{
Z. B. WANG \& Y.W. HUANG \\ Chongqing Academy of Social Sciences, China
}

\begin{abstract}
The sound development of the cultural sphere plays a crucial role in enhancing a country's soft power. Built on the Cultural Competition Index proposed by Meng Dongfang and Wang Zibo (2013,2014), this paper focuses on describing the spatial distribution and concentration relationship of the cultural competition index, taking into consideration of the effects of cultural competition on regional economic development with both spatial and temporal factors. The results obtained from two spatial weight matrixes show that cultural factors play a certain role in promoting local economic growth and have positive spillover effects on the surrounding areas, thus confirming the significant role that the level of cultural competition in promoting the overall economic growth.
\end{abstract}

KEYWORD: Cultural Competition Index; Spatial Correlation; Dynamic Space Panel

\section{INTRODUCTION}

Throughout the human history, culture has been regarded as an extremely important factor by sociologists. Early economists such as Adam Smith, John Muller, and Alfred Marshall had long noted the impact of the cultural factors on society and economy. However, in their theories culture has always been viewed as imposing constraints and norms on people's values in their economic activities. Max Weber pointed out in his book "The Protestant Ethic and the Spirit of Capitalism" that the culture has a decisive role in capitalist development, greatly contributes to the soundness of the market economic system, and is the cornerstone in the development of modern Western economic system. With the improvement of World Values Survey (WVS) data, overseas research related to culture is gradually growing (Guiso, et al., 2006; Maridal, 2013). However, very few studies on culture are related to the economic field. One reason is that the specific mechanism and direction of the impact by cultural factors on economic development and on many other sub-fields cannot be determined, so that it is difficult for economist to find a suitable point to start. The other reason is that cultural factors are ideological in nature and therefore it is difficult to find a specific measure or indicator system to represent culture.

This leads to such a phenomenon: there is not a lack of literature related to culture, but most stays as theoretical debates and of the qualitative nature. Researchers believe that culture has a decisive impact on the micro activity and macro-policy, which gave birth to the exploration of the impact of the national culture on economic and business development models (Zhang \& Gu, 2008). However, empirical studies that use cultural factors as variables are still very scarce. Chou \& Xiao (2011) statistically validated the importance of cultural factors by using micro-survey data; Zhou \& Wu (2010), and Xiong \& Guo (2011) constructed the index system of cultural soft power that can be used to quantitatively compare and analyze the cultural soft power of different regions, providing a basis for the empirical analysis in this study. However, cultural competition index that this study draws on has more explanatory power and policy implications, and the theoretical framework and computational process are more rigorous. Adopting rigorous research methods in economics, and taking into consideration of all factors in the economic system, this study intends to demonstrate the impact of cultural competition on economic development in order to add to the empirical research on cultural competition. It is believed that the analysis drawing on the cultural competition index will lead to an informed conclusion. 


\section{EMPIRICAL MODEL}

How does the cultural competition affect regional economic growth, and how much is the effect? Previous theoretical analysis is far from enough to answer this question, and an appropriate econometric analysis is necessary. First, in this section the economic nature of the cultural competition will be addressed by adopting the cultural competition system and index proposed by Meng \& Wang (2013, 2014), combined with inter-provincial panel data of China's economic development.

The cultural competition index of China's provinces and cities constructed by Meng \& Wang (2013, 2014) has Information on two dimensions: time and space, and has effectively overcome many difficulties such as multicollinearity and time sequence, thus providing more degree of freedom, less collinearity and higher estimate efficiency. The panel data analysis has become a major measurement instrument in empirical analysis. In this study, the general panel model that does not consider spatial effects was selected to analyse the impact of cultural competition on economic development. Specifically the model is set as follows:

$$
y_{i t}=a_{0}+a_{1} C u l_{i t}+\bar{\beta} Z+\mu_{i}+\varepsilon_{i t}
$$

In the equation, $y_{i t}$ is the dependent variable in this paper, indicating the level of economic development of various regions. $C u l_{i t}$ is the core independent variable that the study needs to focus on, and is measured with the cultural competition index that is constructed. $\mathrm{Z}$ stands for a series of control factors that impact on economic development and will be elaborated below. The coefficient before the variable reflects the direction and magnitude of the impact of various factors on the economic development. $\mu_{i}$ is the individual effect that does not change with time, and $\varepsilon_{i t}$ indicates the random error.

\subsection{Index Selection and Data Sources}

1. The level of economic development, expressed with $Y$. In the existing literature on economic development, there are a variety of indicators to measure the level of economic development, such as per capita income, per capita GDP and the corresponding logarithm and so on. By comparison, we believe that the natural logarithm of regional GDP per capita is a better measure because it not only keeps the difference between levels of magnitude in balance, but also reduces more effectively the possibility of heteroscedasticity.

2. Cultural competition, expressed with $\mathrm{Cul}$. To illustrate that the cultural competition also has a significant impact on economic development, the cultural competition index that is constructed will introduced into the model. For specifics regarding the origin and description of the index, please refer to Meng \& Wang $(2013,2014)$.

3. Physical capital stock, expressed with $K$. In classical production function model, the capital stock is one of the most important factors in economic growth and long-term development. The amount of the per capita capital stock in a region directly determines the trend of its economic development. However, Chinese authorities do not provide official data regarding capital stock in different regions, so scholars usually estimate them when they need them. The common method for estimating capital stock is the simplified perpetual inventory method, and the four key variables are selected with different criteria (Zhang et al., 2004; Shan, 2008; Ye, 2010). In this study, we mainly consulted Zhang et al's (2004) estimate for the inter-regional capital stock, whereby the comparable interprovincial capital stock was obtained. For a similar level of economic development, the natural logarithm of the data was obtained.

4. Industrial structure, expressed with Ind. In general, at the early stage of economic development, physical capital and human capital are relatively scarce, the main goal of economic growth is to accelerate the process of industrialization, which will inevitably lead to a rapid increase of the proportion of the manufacturing industry in economy. With the further economic development, the region has entered a post-industrial era, starting to rely on a more value-added high-end service industry. Therefore, the industrial structure is a key indicator of the level of economic development. Based on Li \& Zhou (2009), this study measured the industrial structure between regions using the proportion of the first industry in the national economy.

5. The level of urbanization, expressed with Urban. China is at the critical period of pushing forward the new urbanization in an overall manner. Urbanization and industrialization not only promote and complement each other as well as make greater use of limited public resources in the city, but have also brought crucial impact on rural land reform and sustainable regional economic development. Some scholars believe that the full-scale urbanization will determine how far China's economic development can go. In accordance with the common practice in most of the literature, this study adopted the proportion of non-agricultural population in the total population as the level of urbanization in China's regions.

6. Financial development, expressed with FIR. The importance of financial development in economic 
growth is self-evident. Financial development can bring about a positive impact on economic development by virtue of facilitating transactions, encouraging bank saving, risk management and many other aspects, and inject greater vitality into the economic system and bring in scarce resources. There are generally multiple indicators to measure the level of financial development, such as financial scale, financial efficiency, and financial control. Based on the research topics and data availability, this study took financial efficiency expressed by the ratio of the balance of the non-state sector loans to the total loan balance in different regions as a measure of the level of financial development.

7. Economic openness, expressed with Open. With the furthering of economic globalization, China's important position in the international arena has become increasingly prominent. Economic openness, as an important variable to reflect the level of opening-up, has become one of the important indicators to measure the level of economic development of a region. An open economic system not only benefits the foreign capital investment, the introduction of advanced technology and management philosophy, but can also create a liberal and inclusive economic environment, all of which have particularly significant impact on economic development. Following most researchers' practice, this study defines economic openness as the proportion of the current year's total imports and exports in the GDP.

8. Infrastructure, expressed with Road. Infrastructure, as a precondition for economic development, provides the momentum for the overall and balanced development of the whole society. Many scholars believe that a sound infrastructure not only provides convenience for the local area, but also shortens the distance between regions, reduces costs of transportation and transaction between regions, and achieves the effect of increasing earnings and economic growth. Existing literature generally uses the total (road and railway) mileage or the amount of goods transported as a proxy variable to measure infrastructure. In this study, highway density in different regions, i.e., highway mileage per unit area was adopted as the measure for the level of infrastructure development.

9. The size of government, expressed with Gov. Finally, we also added the size of government as a variable to represent the degree of marketization of the regions. Despite thirty years of reform and opening up which has significantly improved China's marketization, the government's macroeconomic regulation and control is still an important factor that cannot be ignored in analyzing the development trend of China's economy. Following the proposal of Fan Gang's market index, research related to the government system reform has sprung up, which also highlights the importance attached to the size of government in academia. In this paper, the regional government expenditure share of in the GDP was adopted as a measure of the size of government.

Guided by the construction system of cultural competition index, most data of the available indicators are after the year 2005, so the duration under study here is 2005-2012. Most of the empirical data for analysis are available from the "China Statistical Yearbook" and GTA database. Economic indicators that appear alone in value underwent appropriate deflator. Tibet Autonomous Region, due to policy support and lack of data, was excluded from the sample.

\subsection{Spatial Analysis}

Given the fact that China's regional economy and cultural competition have significant spatial nature, ignoring the mechanisms by which spatial factors exert their influence and adopting the ordinary panel model may create some errors in model estimation, so by drawing upon a series of variables in the panel model discussed in the previous section, while introducing the spatial lags of the independent variables and core explanatory variables, the model was set as follows:

$$
\begin{aligned}
& Y_{i t}=\alpha_{0}+\alpha_{1} W_{i j} Y_{i t}+\alpha_{2} \text { Cul }_{i t}+\alpha_{3} W_{i j} \text { Cul }_{i t} \\
& +\beta_{1} K_{i t}+\beta_{2} \text { Ind }_{i t}+\beta_{3} \operatorname{Urban}_{i t}+\beta_{4} \text { FIR }_{i t} \\
& +\beta_{5} \text { Open }_{i t}+\beta_{6} \operatorname{Road}_{i t}+\beta_{7} \text { Gov }_{i t}+\mu_{i}+\varepsilon_{i t}
\end{aligned}
$$

Table 1 shows the estimated results of the spatial panel model under the adjacency weights matrix and distance weights matrix. The goodness of fit of both matrixes is satisfying, there was not much difference between the LogL and LR values, and the estimates under the adjacency matrix are slightly more efficient. The estimation coefficients under both weight matrixes have the same direction, and the difference between the absolute values is within the acceptable range, indicating that the regression results are robust.

Spatial lags of the economic growth are significantly positive, and pass the $1 \%$ significance level test. For every $1 \%$ increase in the economic growth in surrounding areas, the local economic growth can be driven up by $0.4-0.53 \%$, a conclusion consistent with Huang \& $\mathrm{Pu}$ (2011) that "China's regional economy is compartmentalized, and a 'coreedge' spatial features have been developed", indicating a strong spatial dependence in China's regional economic development. At the same time, spatial lags of the cultural competition index is also positive, and at least passes the 5\% significance 
level, indicating that the increase in cultural competition index in surrounding areas has a positive role in promoting the economic growth of the local region. This can be explained as follows: cultural competition is competition of soft power in the ideological sphere between regions, and can be learned and supplemented by microscopic daily exchange and communication between individuals. With the improvement of people's living standards, people pay more attention to the culture consumption and education, and adjacent areas will inevitably compete with each other, thus boosting the concentration of cultural competition in the surrounding area. On the other hand, cultural competition has a strong "ratchet effect", so its impact on economic growth will gradually accumulate in a cyclic manner. The improvement of the local cultural competition is known to positively correlate with the local economic growth, so the cultural competition in the surrounding areas promotes the economic growth in the local region through its effects on the local cultural competition. In addition, the improvement of the cultural competition in the surrounding area also helps to improve the system and regulations of the local region, reduce transaction costs of the local region in building economic relations with the outside world, and promote more reasonable configuration of the regional economic elements, which can also bring about positive spillover effect of the cultural competition.

As for the relevant control variable, physical capital stock has a critical role in promoting economic growth, and the industrial structure variables represented by the first industry has certain inhibition effect on economic growth. In addition, the urbanization process, and improved financial efficiency and infrastructure have also promoted economic growth to varying degrees. The regression result of the openness in this paper was not significant, and government regulation also has a negative impact on economic growth, indicating that China's economic system has not yet fully liberalized, government regulation is still obvious, and the degree of marketization needs improving. The above results are basically the same as arguments in the mainstream economics, and we will not do much to explain.

It should be noted that here the regression was not performed respectively based on the division of the eastern, central and western regions, because firstly, doing so would greatly reduce the sample of the model, resulting in the insignificance or distortion in the model coefficient; secondly the differences within China's regions is much smaller than those between regions, and division of regions would make spatial properties between provinces less significant, and would not meet the basic conditions for the use of spatial measurement. Thus, the panel model estimates of the national sample under only two weights matrixes are conducted to express the relationship between cultural competition and economic development.

Table 1 spatial panel estimation results of the cultural competition effect on economic growth

\begin{tabular}{|c|c|c|}
\hline & $\begin{array}{c}\text { Adjacency weights } \\
\text { matrix }\end{array}$ & $\begin{array}{c}\text { distance weights } \\
\text { matrix }\end{array}$ \\
\hline \multirow{2}{*}{$W Y$} & $0.3958^{* * * *}$ & $0.5301^{* * * *}$ \\
\hline & $(0.0465)$ & $(0.0817)$ \\
\hline \multirow{2}{*}{ Cul } & $0.0489^{\text {**** }}$ & $0.0453^{* * *}$ \\
\hline & $(0.0163)$ & $(0.0229)$ \\
\hline \multirow{2}{*}{ WCul } & $0.0614^{* * *}$ & $0.0568^{* * * *}$ \\
\hline & $(0.0684)$ & $(0.0547)$ \\
\hline \multirow{2}{*}{$K$} & $0.4358^{\text {**** }}$ & $0.5144^{* * *}$ \\
\hline & $(0.0339)$ & $(0.0359)$ \\
\hline \multirow{2}{*}{ Ind } & $-0.0047^{* *}$ & -0.0038 \\
\hline & $(0.0021)$ & $(0.0024)$ \\
\hline \multirow{2}{*}{ Urban } & $0.0035^{* * * *}$ & $0.0024^{* *}$ \\
\hline & $(0.0009)$ & $(0.0012)$ \\
\hline \multirow{2}{*}{ FIR } & $0.0531^{\text {*** }}$ & 0.0157 \\
\hline & $(0.0156)$ & $(0.0181)$ \\
\hline \multirow{2}{*}{ Open } & $0.0014^{* * *}$ & $0.0027^{* * * *}$ \\
\hline & $(0.0003)$ & $(0.0004)$ \\
\hline \multirow{2}{*}{ Road } & 0.0003 & $0.0007^{*}$ \\
\hline & $(0.0003)$ & $(0.0004)$ \\
\hline \multirow{2}{*}{ Gov } & $-0.0078^{* * *}$ & $-0.0138^{* * *}$ \\
\hline & $(0.0013)$ & $(0.0016)$ \\
\hline \multirow{2}{*}{ _cons } & $1.3603^{* * *}$ & $2.3365^{\text {*** }}$ \\
\hline & $(0.3840)$ & $(0.3381)$ \\
\hline$A d j-R^{2}$ & 0.8654 & 0.9367 \\
\hline $\log L$ & 361.4672 & 341.9951 \\
\hline$L R$ & 142.3323 & 136.6547 \\
\hline
\end{tabular}

Notes: $* * *, * *, *$ represent $1 \%, 5 \%, 10 \%$ significance levels respectively; values in parentheses are standard deviations.

\section{A BRIEF CONCLUSION}

This article briefly describes the difficulties in empirical research on the effect of cultural factors on economic development. By drawing on existing research findings and cultural competition index system, the inter-provincial panel data model was constructed. With spatial factors taken into consideration, it finds that China's regional economy and cultural competition present significant spatial concentration, with provinces located on the high or low ends in the cultural competition quartile map are generally distributed in chunks, and this trend becomes more apparent as time went on. Meanwhile, the spatial econometric model shows that not only the cultural competition in the local region promoted economic growth, the improvement of the cultural 
competition in surrounding areas also has a positive spillover effect on the local economic growth, which further confirms that improving the cultural competition plays a significant role in regional economic growth.

\section{REFERENCES}

[1] Maridal, J. H. (2013). Cultural Impact on National Economic Growth, The Journal of Socio-Economics, 47: 136-146.

[2] Guiso, L., Sapienza, P., \& Zingales, L. (2006) Does Culture Affect Economic Outcomes? NBER Working Paper.

[3] Chou, L \& Xiao, R. (2011) Cultural capital and social status attainment - An Empirical Study of Shanghai, China Social Science, (6): 121-135.

[4] Shan, H. (2008). Re-estimation of Chinese capital stock K: 1952-2006, Quantity Technical Economics, (10): 1731.

[5] Huang, S. \& Pu, Y. (2011). Research on spatial mechanism of regional differences causing chunky development of economy, Statistical Research, 28 (4): 4248 .
[6] Li, T. \& Zhou, Y. (2009). Competition among Chinese local government expenditure - Empirical evidence based on Chinese provincial panel data, Management World, (2): 12-22.

[7] Meng, D. \& Wang Z. (2013). Theoretical framework and practical application of China's cultural competition index, Reform, (11): 146-156.

[8] Meng, D. \& Wang Z. (2014). China Cultural Research Outline of competing systems Chongqing University (Social Sciences), 20 (5): 135-140.

[9] De, X. \& Guo. F. (2011). A study on a country's cultural soft power evaluation and promotion approach, China Industrial Economy, (9): 16-26.

[10] Ye, Z. (2010). Chinese provincial capital stock estimates, Statistical Research, (12): 65-71.

[11] Zhang, Y. \& Gu, M. (2008). The dragon and the elephant: A comparative analysis of India's and China's reform and development, Statistical Research, 25 (10): 34-39.

[12] Zhang, J. Wu, G., \& Zhang, J. (2004). China's provincial capital stock estimates: 1952-2000, Economic Research, (10): 35-44.

[13] Zhou, G. \& Wu, D. (2010). A comparative study of cultural soft power between China's provinces, Statistical Research, 27 (2): 7-14. 